\title{
Crohn Disease-Like Reaction
}

National Cancer Institute ( $\mathrm{NCl})$

\section{Source}

National Cancer Institute (NCI). Crohn Disease-Like Reaction.

Discrete aggregates of lymphoid white blood cells, some with germinal centers and surrounding fibrosis, commonly found around some colorectal adenocarcinomas in the absence of a clinical or pathological diagnosis of Crohn disease. Also called Crohn-like reaction. 